\title{
Covid-19 Impact on Macular Neovascularization and Retinal Vein Occlusion Treatment: Single-Center Experience
}

\author{
Rodrigo Vilares-Morgado ${ }^{a, b, c}$ Carolina Madeira ${ }^{a} \quad$ Ana Maria Cunha ${ }^{a}$ \\ Manuel Falcãoa,b,c João Beato ${ }^{a}$ Ana Catarina Pedrosa ${ }^{a, c}$ Susana Penas ${ }^{a, c}$ \\ Elisete Brandão ${ }^{a, c}$ Fernando Falcão-Reis ${ }^{a, b, c}$ Ângela Carneiro ${ }^{a, b, c}$ \\ aDepartment of Ophthalmology, Centro Hospitalar de São João Hospital, Porto, Portugal; bepartment of Surgery \\ and Physiology, Faculty of Medicine of University of Porto, Porto, Portugal; 'Faculty of Medicine of Porto University, \\ Porto, Portugal
}

\section{Keywords}

COVID-19 - Antivascular endothelial growth factor treatment · Macular neovascularization · Retinal vein occlusion

\begin{abstract}
Purpose: The aim of this study was to evaluate whether the coronavirus disease 19 (COVID-19) pandemic resulted in undertreatment and subsequent loss of visual acuity (VA) in patients with macular neovascularization (MNV) or retinal vein occlusion (RVO) regularly treated with intravitreal antivascular endothelial growth factor injections. Methods: Single-center, retrospective study of patients scheduled for treatment between March 19 and June 1, 2020, the national mandatory quarantine period. Patients' demographics, VA, and scheduled treatment during this period were reviewed via medical records. All patients were analyzed regarding treatment attendance rates. The visual impact of COVID-19 was assessed in patients who had been treated and presented a stable VA for $>6$ months before the beginning of the quarantine. Results: This study included 927 eyes from 769 patients. The attendance rate increased throughout the study timeframe $(p<0.001)$ and correlated negatively with higher patient's age $(r=-0.142 ; p=$ $0.005)$. Patients with age-related macular degeneration (67.6\%)
\end{abstract}

karger@karger.com www.karger.com/bmh

Karger $\stackrel{\text { ' }}{5}$

GOPEN ACCESS
(C) 2021 The Author(s)

Published by S. Karger AG, Basel

This is an Open Access article licensed under the Creative Commons Attribution-NonCommercial-4.0 International License (CC BY-NC) (http://www.karger.com/Services/OpenAccessLicense), applicable to the online version of the article only. Usage and distribution for commercial purposes requires written permission. had lower attendance rates $(p=0.007)$ and were older $(p<$ 0.001). The visual impact analysis included 400 eyes from 325 patients. The average VA variation throughout this period was $-1.7 \pm 8.4$ ETDRS letters and was similar in different retinal pathologies $(p=0.334)$. VA variation did not correlate with the number of missed treatments per patient $(r=0.100 ; p=0.150)$. The prevalence of subretinal fluid and intraretinal fluid, as well as central retinal thickness decreased significantly throughout the study period ( $p$ values of $<0.001,<0.001$, and 0.032 , respectively). Conclusion: The COVID-19 pandemic had a significant impact on the attendance rate of patients with MNV or RVO to their scheduled treatments, which was higher in the first week of mandatory quarantine. Nevertheless, VA did not decrease significantly during this period, with a limited VA variation regardless of primary retinal disorder and morphological parameters even improved in the eyes included in the visual impact analysis.

(c) 2021 The Author(s)

Published by S. Karger AG, Basel

\section{Introduction}

Macular neovascularization (MNV) consists of an abnormal growth of vessels from the choroidal or the retinal circulation that invades the outer retina, subretinal space, 
or sub-RPE space in varying combinations [1]. The balance between intraocular antiangiogenic factors and angiogenic factors dictates the growth of these new vessels. The most potent intraocular angiogenic factor is vascular endothelium growth factor (VEGF) [2]. Therefore, the current treatment of MNV consists of regular intravitreal injections of anti-VEGF drugs [3]. This treatment significantly improves functional and morphological outcomes in MNV from multiple etiologies [4], leading to significantly long-term higher visual acuity (VA), when compared to older treatment modalities $[4,5]$. To prevent deterioration of VA, patients are generally willing to accept a high treatment burden with regular intravitreal injections at short intervals [6]. Nevertheless, the injection burden for healthcare systems is significant, and some patients might be undertreated. Recent studies have validated alternative treatment regimens for neovascular age-related macular degeneration (AMD) and other causes of MNV that reduce the clinical burden of antiVEGF treatment, while maintaining good functional results $[7,8]$. Macular edema (ME) is also an important cause of visual impairment in high-income countries. In both diabetic retinopathy (DR) and retinal vein occlusion (RVO), the two most frequent retinal vascular disorders [3], ME is the main cause of vision loss. Treatment of ME secondary to RVO also relies significantly on anti-VEGF injections with all of their benefits and costs $[9,10]$. As in MNV, alternative treatment regimens for ME secondary to RVO have been investigated recently, with the objective of reducing the anti-VEGF injection burden [11].

The coronavirus disease 19 (COVID-19) had a significant impact in every aspect of modern societies. COVID-19 was declared a pandemic on March 11, 2020, by the World Health Organization (WHO). All around the world, healthcare changed dramatically. The pandemic reinforced the need to distance physically, to minimize nonessential exposure, to acquire appropriate personal protective equipment for both providers and patients, to enhance sanitization and hygiene practices, and to cope with the additional costs of doing so [12-14]. In Portugal, our government and national public health department implemented a period of mandatory nationwide quarantine that begun on the 19th of March and ended on the 1 st of June. In healthcare, numerous adjustments were made to protect the health of our general population and our healthcare workers [15]. These included transitioning to virtual medical consultations when possible. Telemedicine in ophthalmology was already used in the past for the detection of DR, for example, with good overall diagnostic accuracy [16]. In our Ophthalmology Depart- ment, we followed the recommendations of the American Academy of Ophthalmology for this new clinical reality. We kept our emergency room available for urgent situations, but we carried out programmed medical consultations via phone call to reduce the elevated number of patients that we have in our department on a regular basis. However, a significant number of patients still required their regular anti-VEGF injection to maintain their disease controlled. This injection had to be given in the safest manner possible. Thus, we changed the venue of the injections to a different part of our hospital which was only being used as an ophthalmology operating room (with its own entrance and exit) and reduced the total number of injections per day by around 5 injections per day, which allowed us to create a bigger interval and implement a more thorough cleaning of the operating room between patients.

The purpose of this study is to determine the impact that COVID-19 had on patients with MNV and/or RVO complicated by ME, more specifically, whether this pandemic resulted in a significant decrease in VA in patients who had their disease controlled prior to this pandemic. Furthermore, we wanted to analyze the attendance rate for anti-VEGF injections during the national quarantine period in patients with MNV and ME due to RVO that had already received multiple injections previously and assess if the reduced total number of injections per day that we carried out led to undertreatment of these patients.

\section{Materials and Methods}

We conducted a single-center, retrospective longitudinal observational study of patients that were scheduled to be treated with anti-VEGF injections for MNV secondary to AMD, central serous chorioretinopathy (CSCR), and myopic maculopathy or ME secondary to branch retinal vein occlusion and central vein retinal occlusion, between March 19, 2020 and June 1, 2020, the Portuguese national mandatory quarantine period. This study took place in Centro Hospitalar Universitário S. João (Porto, Portugal), a tertiary university hospital that serves the northern region of Portugal. Research adhered to tenets of the Declaration of Helsinki and approval for this study was obtained from the Ethics Committee of the hospital.

Patients were identified through a search of an electronic calendar available at our Retina subdepartment for scheduling intravitreal injections. All patients scheduled to be treated during this period were analyzed regarding treatment adherence and persistence. Their medical records were reviewed to determine the number of treatments scheduled and the number of treatments they actually received during the study period, and the attendance rate was calculated. The visual impact of the COVID-19 pandemic was assessed only in patients with stable disease before the be-
Vilares-Morgado et al. 
Table 1. Anti-VEGF injections scheduled for the national mandatory quarantine period that lasted a total of 11 weeks and proportion of patients of attended and missed their appointed treatments

\begin{tabular}{llll}
\hline Week of quarantine period & \multicolumn{2}{l}{ Scheduled treatments } & total \\
\cline { 2 - 4 } & $\begin{array}{l}\text { missed } \\
\text { (\% of weekly total) }\end{array}$ & $\begin{array}{l}\text { attended } \\
\text { (\% of weekly total) }\end{array}$ \\
\hline 1st (16-20 Mar) & $53(42.7)$ & $71(57.3)$ & 124 \\
2nd (23-27 Mar) & $52(45.6)$ & $62(54.4)$ & 114 \\
3rd (30 Mar to 3 Apr) & $55(45.8)$ & $65(54.2)$ & 120 \\
4th (6-10 Apr) & $41(39.8)$ & $62(60.2)$ & 103 \\
5th (13-17 Apr) & $39(30.7)$ & $88(69.3)$ & 127 \\
6th (20-24 Apr) & $53(31.5)$ & $115(68.5)$ & 168 \\
7th (27 Apr to 1 May) & $28(23.9)$ & $89(76.1)$ & 117 \\
8th (4-8 May) & $36(23.8)$ & $115(76.2)$ & 151 \\
9th (11-15 May) & $28(17.2)$ & $112(86.8)$ & 163 \\
10th (18-22 May) & $17(13.2)$ & $103(83.1)$ & 124 \\
11th (25-29 May) & $21(16.9)$ & $1,017(70.6)$ & 1,440 \\
\hline Total & $423(29.4)$ & &
\end{tabular}

Throughout this period, the number and proportion of patients who attended scheduled treatments gradually increased and the number and proportion of patients who did not attend their scheduled treatments progressively decreased $(p<0.001)$. VEGF, vascular endothelium growth factor. ginning of the study period, that is, patients who presented and were treated for MNV and/or ME for $>6$ months and who presented a stable VA (with a variation of under 5 ETDRS letters) also during the last 6 months before the onset of the aforementioned period. These patients' medical records were reviewed, and data regarding their demographic information, VA using Early Treatment Diabetic Retinopathy Study (ETDRS) chart, and type and number of treatments scheduled and received during this period were collected. Tomographic data of the eyes included in the visual impact analysis were also gathered using the electronic database available at our SPECTRALIS Heidelberg ${ }^{\circledR}$ Retina Angiograph (HRA) + Optical Coherence Tomography (OCT) imaging platform (Heidelberg Engineering, Heidelberg, Germany). We determined the presence of subretinal fluid (SRF), intraretinal fluid (IRF), and central retinal thickness (CRT), that is, retinal thickness in the central $1 \mathrm{~mm}^{2}$ of the acquired central volume scans in the different time points. Data were collected at three different time points: at diagnosis of exudative MNV and/or ME, at the last medical consultation before the onset of the study period, and at the first medical consultation after the aforementioned period. We excluded patients receiving anti-VEGF treatment due to diabetic ME, patients with controlled disease who did not require continuous treatment during the whole quarantine period and patients who had already missed $\geq 2$ consecutive injections previously. Furthermore, patients without medical follow-up after the quarantine period were also excluded from the visual impact of COVID-19 analysis.

Afterward, we determined the patient attendance rate for intravitreal treatments in each week of the study period and the total and per week patient attendance rate according to the primary retinal disorder, analyzing their evolution through time in all patients scheduled to be treated. The attendance rate was calculated for each patient and not for each eye. We performed a $\chi^{2}$ test to correlate the attendance rate with the week of the study period and to correlate the attendance rate with primary retinal disorder. We performed a Pearson correlation to correlate the attendance rate with patients' age. To determine the visual impact the COVID-19 pandemic had on patients with stable disease, we determined average VA variation (difference between VA at the last medical consultation before beginning of the study period and VA at the first medical consultation after completion of the mandatory quarantine period) using the ETDRS chart. In the Retina subdepartment of our Ophthalmology Department, we measure VA with the ETDRS scale. Therefore, all presented VA determinations were originally measured with the ETDRS scale and no conversion was necessary. VA variation was calculated for each eye and not for each patient. We then performed a subgroup analysis to determine VA variation according to the primary retinal disease, VA variation according to the number of missed treatments during this period, and VA variation according to the follow-up time before the beginning of the national mandatory quarantine period. Finally, we characterized the subgroup of patients with a significant VA reduction, defined as a decrease in VA of 5 or more ETDRS letters.

Normally distributed data are reported as mean and standard deviation (SD), while non-normally distributed data are reported as median and interquartile range. Kolmogorov-Smirnov and Shapiro-Wilk tests were used to assess whether each variable followed a normal distribution. Comparisons between groups were performed using independent samples $T$ test (for normally distributed data) or Mann-Whitney U test (for non-normally distributed data) for continuous variables, while the $\chi^{2}$ test was used for categorical variables. $p$ value $<0.05$ was considered statistically significant. For statistical analysis, SPSS Statistics 26 was used. 
Table 2. Patients who attended and missed their scheduled intravitreal injections during the study period, grouped according to their retinal disorder

\begin{tabular}{llll}
\hline Retinal disorder & \multicolumn{3}{l}{ Scheduled treatments } \\
\cline { 2 - 4 } & $\begin{array}{l}\text { missed } \\
\text { (\% of total) }\end{array}$ & $\begin{array}{l}\text { attended } \\
\text { (\% of total) }\end{array}$ & total \\
\hline AMD & $310(32.2)$ & $646(67.8)$ & 1,011 \\
CRVO and BRVO & $68(24.6)$ & $208(75.4)$ & 276 \\
Myopic maculopathy & $15(17)$ & $73(83)$ & 88 \\
CSCR & $14(21.5)$ & $51(78.5)$ & 65 \\
Total & $423(29.4)$ & $1,017(70.6)$ & 1,440 \\
\hline
\end{tabular}

There is a statistically significant difference in attendance rate according to baseline retinal disorder $(p=0.002)$. AMD, age-related macular degeneration; CSCR, central serous chorioretinopathy; CVRO, central vein retinal occlusion; BRVO, branch retinal vein occlusion.

\section{Results}

\section{Attendance Rate Analysis}

Attendance rate per week data are presented in $\mathrm{Ta}$ ble 1 and attendance rate according to retinal disease data are presented in Table 2. A total of 1,735 intravitreal anti-VEGF injections were scheduled for the 11-week period of national mandatory quarantine. These included 927 eyes from 769 patients (463 female and 306 male) with a mean age of $77.7 \pm 11.2$ years. Patients with AMD had a mean age of $80.9 \pm 8.0$ years, patients with RVO had a mean age of $74.5 \pm 11.8$ years, patients with myopic maculopathy had a mean age of $57.5 \pm 14.3$ years, and patients with CSCR had a mean age of $71.9 \pm 15.3$ years. Therefore, patients with AMD were significantly older than patients with other retinal disorders $(p<0.001)$. A total of 1,218 anti-VEGF injections were administered. The overall attendance rate was $70.6 \%$ and correlated negatively with higher patient's age $(r=-0.142 ; p=$ $0.005)$. The attendance rate increased significantly throughout the study timeframe $(p<0.001)$, from $57.3 \%$ in the first week and $54.4 \%$ in the second week to $83.1 \%$ in the last week of the quarantine period. Thirty-one patients $(4.03 \%)$ were lost to follow-up, missing all scheduled treatments and subsequent medical consultations. 669 eyes $(72.2 \%)$ presented AMD, 167 eyes (18\%) presented RVO, 53 eyes (5.7\%) presented myopic maculopathy, and 38 eyes (4.1\%) presented CSCR. Patients with AMD (67.8\%) had lower attendance rates than patients with branch retinal vein occlusion/central vein retinal occlusion $(75.4 \%)$, myopic maculopathy $(83.0 \%)$, or
CSCR $(78.5 \%)(p=0.002)$. One case of COVID-19 was confirmed in one of the study patients during the study period.

\section{Visual Impact of the COVID-19 Pandemic Analysis}

The visual impact analysis included 400 eyes from 325 patients (196 females and 129 males). Average follow-up since MNV and/or ME diagnosis was $65 \pm 42$ months. 269 (67.6\%) eyes were scheduled to be treated every 3-6 weeks, $112(28.1 \%)$ eyes were scheduled to be treated every 7-10 weeks, and 17 eyes (4.3\%) were scheduled to be treated every 11-14 weeks. Interval treatments were similar in eyes with different retinal pathologies $(p=0.265)$. In all primary retinal disorders, the majority of the studied eyes were scheduled to be treated every 3-6 weeks. The attendance rate also did not correlate with treatment intervals $(r=-0.02 ; p=0.691)$.

A treat-and-extend regimen was used in almost all studied eyes (97.5\%). Eight-five percent of the eyes were initially injected with bevacizumab, $8.5 \%$ were initially injected with ranibizumab, and $6.5 \%$ were initially injected with aflibercept. Throughout follow-up since MNV and/or ME diagnosis, 207 eyes (51.7\%) had their anti-VEGF drug switched, mostly to aflibercept (89.9\%), but also to bevacizumab (3.4\%) and ranibizumab (6.3\%).

During the study period, $50.6 \%$ of the eyes did not miss any scheduled injection, $25.8 \%$ of the eyes missed 1 scheduled injection, $16.0 \%$ of the eyes missed 2 scheduled injections, and $7.5 \%$ of the eyes missed 3 scheduled injections. Average VA before the beginning of the quarantine period was $53.6 \pm 19.9$ ETDRS letters in eyes with AMD, $49.4 \pm 26.7$ ETDRS letters in eyes with RVO, $54.2 \pm 16.5$ ETDRS letters in eyes with myopic maculopathy, and 62.7 \pm 18.7 ETDRS letters in eyes with CSCR.

A histogram of VA variation is depicted in Figure 1. The average VA variation during this period was $-1.7 \pm$ 8.4 ETDRS letters, with a maximum decrease of 38 ETDRS letters and a maximum increase of 47 ETDRS letters in VA. The average VA variation was $-2.4 \pm 7.9$ ETDRS letters in eyes with AMD, $-0.5 \pm 8.2$ ETDRS letters in eyes with RVO, $1.0 \pm 13.5$ ETDRS letters in eyes with myopic maculopathy, and $-0.4 \pm 5.4$ ETDRS letters in eyes with CSCR. Thus, the average VA variation was similar in different retinal pathologies $(p=0.334)$. Neither age $(r=-0.114 ; p=0.092)$ nor number of treatments missed per patient $(r=0.100 ; p=0.150)$ correlated with VA variation. VA variation was also similar in patients with a follow-up of 12 months or less since MNV and/or $\mathrm{ME}$ diagnosis and patients with a follow-up of more than 
Fig. 1. VA variation throughout the study period. First available VA after conclusion of the national quarantine period was subtracted from the last available VA before the onset of the aforementioned period. The average VA variation was $-1.67 \pm 8.4$ ETDRS letters. VA, visual acuity; ETDRS, Early Treatment Diabetic Retinopathy Study.

Table 3. Tomographic data of the eyes included in the visual impact of the COVID-19 analysis

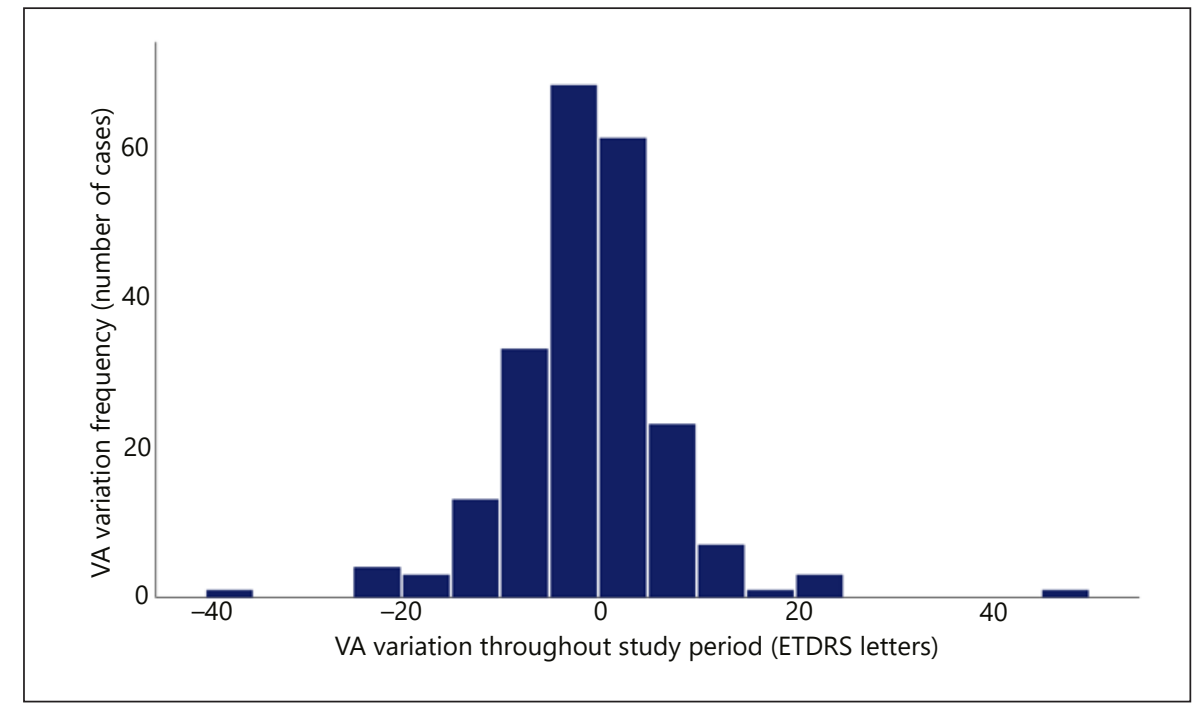

\begin{tabular}{|c|c|c|c|}
\hline & $\begin{array}{l}\text { Before Portuguese national } \\
\text { mandatory quarantine period } \\
(N=394)\end{array}$ & $\begin{array}{l}\text { After Portuguese national } \\
\text { mandatory quarantine period } \\
(N=359)\end{array}$ & $p$ value \\
\hline Presence of SRF, $n(\%)$ & $99(25.1)$ & 70 (19.5) & $<0.001$ \\
\hline Presence of IRF, $n(\%)$ & $115(29.2)$ & $79(22.0)$ & $<0.001$ \\
\hline CRT, $\mu \mathrm{m}$ & $304.77 \pm 95.49$ & $296.40 \pm 92.46$ & 0.032 \\
\hline
\end{tabular}

Values for CRT are presented as mean \pm standard deviation. SRF, subretinal fluid; IRF, intraretinal fluid; CRT, central retinal thickness.
12 months since MNV and/or ME diagnosis ( $p=0.643$ ). In addition, follow-up since MNV and/or ME diagnosis did not correlate with VA variation $(r=-0.019 ; p=$ 0.776).

Regarding tomographic data, which are present in Table 3, 99 eyes (25.1\%) presented SRF in the optical coherence tomography performed before the study period, while 70 eyes $(19.5 \%)$ presented SRF after the study pe$\operatorname{riod}(p<0.001)$. The proportion of eyes with IRF also decreased significantly throughout the study period (115 eyes or $29.2 \%$ before vs. 79 eyes or $22 \%$ after the study period; $p<0.001)$. CRT decreased from $304.77 \pm 95.49$ $\mu \mathrm{m}$ to $296.40 \pm 92.46 \mu \mathrm{m}(p=0.032)$. Out of the 105 eyes with IRF before the study period with available tomographic data after the study period, 43 eyes $(41 \%)$ presented no IRF after the study period $(p<0.001)$, while out of the 93 eyes with SRF before the study period and available tomographic data after the study period, 37 eyes (39.8\%) presented no SRF after the study period $(p<$ $0.001)$. Only 17 eyes $(4.74 \%)$ presented de novo SRF or IRF after the study period.
As for eyes with significant VA loss (66 eyes or $30.3 \%$ of our sample), defined as a decrease in VA of 5 or more ETDRS letters, this subgroup consisted mainly of eyes with AMD (74.1\%) and RVO (18.5\%). Not a single eye with CSCR presented such a negative variation of VA in this period. Most eyes (66.7\%) were scheduled to be treated every 3-6 weeks. The average patient age was $78.9 \pm$ 11.5 years. The average follow-up since MNV and/or ME diagnosis was $58 \pm 43$ months. $51.9 \%$ of the eyes with this significant $V A$ reduction had no missed anti-VEGF injections, $33.3 \%$ missed 1 injection, and $14.8 \%$ missed 2 or more injections during the study period. Significant VA loss did not correlate with the presence of SRF or IRF de novo after the study period $(p=0.115)$. Comparing eyes with significant VA loss with eyes without significant VA loss, there were no significant differences in patient's age $(p=0.318)$, primary retinal disease $(p=0.481)$, treatment interval ( $p=0.993)$, number of missed anti-VEGF treatments $(p=0.151)$, or follow-up since MNV and/or ME diagnosis $(p=0.574)$. 


\section{Discussion}

In our cohort of patients with MNV and/or ME being actively treated with anti-VEGF injections before, during, and after the national COVID-19 mandatory quarantine period, the attendance rate to scheduled injection increased significantly from the 1st week to the 11 th and final week of the aforementioned period. Patients with AMD had lower attendance rates than patients with other retinal diseases included in this study. In spite of the reduction in attendance rate to scheduled intravitreal injections caused by the COVID-19 pandemic, VA variation was limited to $-1.7 \pm 8.4$ ETDRS letters during this period and was similar regardless of patient's age, primary retinal disorder, treatment interval, number of missed treatments, and follow-up length.

COVID-19 had a profound impact on our everyday lives and complicated healthcare administration to a point that we are just beginning to comprehend [17]. When it comes to ophthalmology departments of high-income countries, there are a large number of patients with exudative retinal disorders, either with accompanying MNV and/or ME, that are being treated with intravitreal anti-VEGF injections. The sustained efficacy of this treatment depends on repeated and regular treatment until disease activity has ceased [3]. The increased visit frequency related to anti-VEGF agent administration has created service pressures on ophthalmology clinics and placed a significant burden on patients and their caregivers, with barriers to treatment adherence and persistence that include patient anxiety/discomfort, financial burden, time constraints, and lack of transportation [6]. The COVID-19 pandemic was an added barrier to this regular treatment and aggravated all these previously existing constraints [18].

Therefore, it does not come as a surprise that when the Portuguese government implemented a national mandatory quarantine period, patients were reluctant to maintain regular visits to their ophthalmology clinic and, as a consequence, attendance rates for scheduled intravitreal injections decreased to as low as $54.4 \%$ in the second week of the study period. To prevent an even more significant decrease in attendance rates, we regularly remembered patients of the need to continue their regular treatment via phone call. We resumed presential medical consultations gradually by the end (around the 10th week) of the quarantine period. This healthcare administration measures, along with a better control of the pandemic in our country, might have contributed to the gradual increase in attendance rates that was observed in our cohort $(p<0.001)$. The fact that patients with AMD had a lower attendance rate for scheduled intra- vitreal injections than patients with other retinal disorders $(p=0.002)$ might be associated with the fact that these patients were also older $(p<0.001)$, since the attendance rate correlated negatively with higher patient's age $(r=-0.142$; $p=0.005)$ in our overall attendance rate analysis.

Nevertheless, the impact of the COVID-19 pandemic on VA of patients with MNV and/or ME due to AMD, RVO, myopic maculopathy, and CSCR was limited to an average VA variation of $-1.7 \pm 8.4$ ETDRS letters. Whether this implies that there was no undertreatment of these patients is still uncertain, since this short period of time might not be sufficient to determine the extent to which the pandemic impacted visual function in patients with exudative retinal disorders. In this study, we could not establish significant correlations with VA variation during this period. Age $(r=-0.114 ; p=0.092)$, number of treatments missed per patient $(r=0.100 ; p=0.150)$, and follow-up since MNV and/or ME diagnosis did not correlate with VA variation $(r=-0.019 ; p=0.776)$ and VA variation was similar in eyes with different retinal diseases $(p=0.334)$. However, we had a nonresidual (4.04\%) percentage of patients that were completely lost to followup, patients in whom more significant reductions in VA may have occurred and that could impact our analysis.

The morphological impact of the early COVID-19 pandemic on patients with MNV and/or ME due to AMD, RVO, myopic maculopathy, and CSCR was also limited. There was a residual percentage $(4.74 \%)$ of eyes with de novo SRF or IRF after the Portuguese national mandatory quarantine period and CRT even decreased significantly during this period $(p=0.032)$. The prevalence of SRF and IRF also decreased significantly, as previously stated $(p<$ 0.001 ). Although these morphological data suggest that our sample of eyes with MNV and/or ME improved during the study period, this improvement is not evident if we consider the visual data. The authors hypothesize that, since we only included eyes with stable disease before the beginning of the study period (eyes who presented and were treated for MNV and/or ME for more than 6 months and who presented a stable VA, with a variation of under 5 ETDRS letters also during the last 6 months before the study period), even if these eyes presented a morphological improvement, this did not result in a visual improvement due to the fact that photoreceptors and the retinal pigment epithelium were already compromised as part of the underlying retinal disease. Nonetheless, our morphological results corroborate the notion that there was no undertreatment of these patients during the study period.

When characterizing eyes with significant VA loss (defined as a VA reduction of over 5 ETDRS letters), we found 
that $74.4 \%$ of those eyes suffered from AMD and $66.7 \%$ of those eyes were scheduled to be treated every 3-6 weeks, indicating eyes that still have significant disease activity. Still, considering that $72.2 \%$ of the eyes that were analyzed in this study suffered from AMD and that $67.6 \%$ of all eyes were scheduled to be treated in the same interval, this prevalence of AMD and treatment interval in eyes with significant VA loss is neither relevant nor a predictive factor for significant VA loss. We did not expect $51.9 \%$ of eyes with significant VA loss to have no missed intravitreal injections, nor that significant VA loss did not correlate with the presence of SRF or IRF de novo after the study period. Since we are looking at eyes with theoretical stable retinal disease (being treated for more than 6 months and having a stable VA in the 6 months that preceded the beginning of the study period), we hypothesize that the reason for significant VA reduction in these eyes might be related to ocular diseases other than their primary retinal disorder. We had isolated cases of significant VA reduction in eyes with epiretinal membranes that progressed during the study period, for example.

The results we present imply that the healthcare organizational measures we implemented at the beginning of the COVID-19 pandemic in our ophthalmology department did not result in a significant undertreatment of patients with MNV and/or ME that had their retinal disease controlled prior to the onset of the pandemic. For now, this is a valid interpretation given the results we obtained. However, further studies are still necessary to validate this statement, since we are evaluating VA variation only 12 months after the beginning of the COVID-19 pandemic in Portugal. Furthermore, this is a retrospective study in which some variables were collected from data available in medical charts. Finally, since we did not possess VA data for patients with DR being treated with anti-VEGF regimens, we could not include this large cohort of patients in our study. Those are the main limitations of our study.

\section{Conclusion}

The COVID-19 pandemic had a significant impact on the attendance rate of patients with MNV (secondary to AMD, myopic maculopathy, and CSCR) or ME (secondary to RVO) to their scheduled treatments, which was higher in the first weeks of national mandatory quarantine, decreasing as time went by and the pandemic became more controlled. Patients with AMD had a lower attendance rate and were older than patients with other primary retinal disorders. Nevertheless, VA did not decrease significantly during this period, with a limited VA variation in patients with different retinal pathologies and morphological parameters even improved in the eyes included in the visual impact analysis.

This study provides new insights on the impact the COVID-19 pandemic had on patients with regular antiVEGF treatments. Nonetheless, further studies that include patients with longer follow-up after the onset of the COVID-19 pandemic are needed to confirm or invalidate our assessments.

\section{Acknowledgments}

This manuscript does not include any nonauthor contributors to acknowledge.

\section{Statement of Ethics}

This study adhered to tenets of the Declaration of Helsinki and was approved by the Ethics Committee of Centro Hospitalar Universitário S. João - reference number CESCHSJ12-17. All subjects included in this study provided written informed consent to participate in this study, enabling clinical research with their clinical data and publication of clinical investigations that included their data (including publication of images).

\section{Conflict of Interest Statement}

The authors have no conflicts of interest to declare.

\section{Funding Sources}

None of the authors have received any contribution for this submission.

\section{Author Contributions}

Rodrigo Vilares Morgado - ophthalmologist - collected clinical data, redacted the manuscript, and contributed to its revision. Carolina Madeira, João Beato, Ana Catarina Pedrosa, Susana Penas, and Elisete Brandão - ophthalmologists - also collected clinical data and contributed to the revision of the manuscript. Ana Maria Cunha, Manuel Falcão, and Fernando Falcão-Reis - ophthalmologists - contributed to the redaction and revision of the manuscript. Ângela Carneiro - ophthalmologist - developed the clinical investigation hypothesis, designed the study methodology, contributed to the redaction and revision of the manuscript, and approved the final version of the manuscript.

\section{Data Availability Statement}

All data generated or analyzed during this study are included in this article and was compiled in a database that is readily available and can be published in a repository if required. Further inquiries can be directed to the corresponding author. 


\section{References}

1 Spaide RF, Jaffe GJ, Sarraf D, Freund KB, Sadda SR, Staurenghi G, et al. Consensus nomenclature for reporting neovascular age-related macular degeneration data: consensus on Neovascular Age-Related Macular Degeneration Nomenclature Study Group. Ophthalmology. 2020;127(5):616-36.

2 Ferrara N, Gerber HP, LeCouter J. The biology of VEGF and its receptors. Nat Med. 2003;9(6):669-76.

3 Brand CS. Management of retinal vascular diseases: a patient-centric approach. Eye. 2012;26 Suppl 2(Suppl 2):S1-16.

4 Carneiro AM, Silva RM, Veludo MJ, Barbosa A, Ruiz-Moreno JM, Falcão MS, et al. Ranibizumab treatment for choroidal neovascularization from causes other than age-related macular degeneration and pathological myopia. Ophthalmologica. 2011;225(2):81-8.

5 Finger RP, Daien V, Eldem BM, Talks JS, Korobelnik JF, Mitchell P, et al. Anti-vascular endothelial growth factor in neovascular agerelated macular degeneration - a systematic review of the impact of anti-VEGF on patient outcomes and healthcare systems. BMC Ophthalmol. 2020;20(1):294.

6 Mueller S, Agostini H, Ehlken C, BauerSteinhusen U, Hasanbasic Z, Wilke T. Patient preferences in the treatment of neovascular age-related macular degeneration: a discrete choice experiment. Ophthalmology. 2016; 123(4):876-83.
7 Mantel I, Niderprim SA, Gianniou C, Deli A, Ambresin A. Reducing the clinical burden of ranibizumab treatment for neovascular agerelated macular degeneration using an individually planned regimen. Br J Ophthalmol. 2014;98(9):1192-6.

8 Eichenbaum DA, Duerr E, Patel HR, Pollack SM. Monthly versus treat-and-extend ranibizumab for diabetic macular edema: a prospective, randomized trial. Ophthalmic Surg Lasers Imaging Retina. 2018;49(11):e191-7.

9 Callizo J, Atili A, Striebe NA, Bemme S, Feltgen $\mathrm{N}$, Hoerauf $\mathrm{H}$, et al. Bevacizumab versus bevacizumab and macular grid photocoagulation for macular edema in eyes with nonischemic branch retinal vein occlusion: results from a prospective randomized study. Graefes Arch Clin Exp Ophthalmol. 2019; 257(5):913-20

10 Feltgen N, Hattenbach LO, Bertelmann T, Callizo J, Rehak M, Wolf A, et al. Comparison of ranibizumab versus dexamethasone for macular oedema following retinal vein occlusion: 1-year results of the COMRADE extension study. Acta Ophthalmol. 2018;96(8): e933-41.

11 Larsen M, Waldstein SM, Priglinger S, Hykin $\mathrm{P}$, Barnes E, Gekkieva M, et al. Sustained benefits from ranibizumab for central retinal vein occlusion with macular edema: 24 -month results of the CRYSTAL Study. Ophthalmol Retina. 2018;2(2):134-42.
12 Ahmed IIK, Hill WE, Arshinoff SA. Bilateral same-day cataract surgery: an idea whose time has come \#COVID-19. Ophthalmology. 2021;128(1):13-14.

13 Hadayer A, Zahavi A, Livny E, Gal-Or O, Gershoni A, Mimouni K, et al. Patients wearing face masks during intravitreal injections may be at a higher risk of endophthalmitis. Retina. 2020;40(9):1651.

14 Felfeli T, Mandelcorn ED. Assessment of simulated respiratory droplet spread during an ophthalmologic slitlamp examination. JAMA Ophthalmol. 2020;138(10):1099-101.

15 Papoutsi E, Giannakoulis VG, Ntella V, Pappa S, Katsaounou P. Global burden of COVID-19 pandemic on healthcare workers. ERJ Open Res. 2020;6(2):00195-2020.

16 Shi L, Wu H, Dong J, Jiang K, Lu X, Shi J. Telemedicine for detecting diabetic retinopathy: a systematic review and meta-analysis. $\mathrm{Br} \mathrm{J}$ Ophthalmol. 2015;99(6):823-31.

17 Al-Anzi BS, Alenizi M, Al Dallal J, Abookleesh FL, Ullah A. An overview of the world current and future assessment of novel COVID-19 trajectory, impact, and potential preventive strategies at healthcare settings. Int J Environ Res Public Health. 2020;17(19):7016.

18 Lai THT, Tang EWH, Chau SKY, Fung KSC, Li KKW. Stepping up infection control measures in ophthalmology during the novel coronavirus outbreak: an experience from Hong Kong. Graefes Arch Clin Exp Ophthalmol. 2020;258(5):1049-55. 